\title{
OPEN Exploring the effectiveness of a COVID-19 contact tracing app using an agent-based model
}

\author{
Jonatan Almagor ${ }^{D}$ \& Stefano Picascia $\mathbb{D}{ }^{\circledR}$
}

A contact-tracing strategy has been deemed necessary to contain the spread of COVID-19 following the relaxation of lockdown measures. Using an agent-based model, we explore one of the technology-based strategies proposed, a contact-tracing smartphone app. The model simulates the spread of COVID-19 in a population of agents on an urban scale. Agents are heterogeneous in their characteristics and are linked in a multi-layered network representing the social structure-including households, friendships, employment and schools. We explore the interplay of various adoption rates of the contact-tracing app, different levels of testing capacity, and behavioural factors to assess the impact on the epidemic. Results suggest that a contact tracing app can contribute substantially to reducing infection rates in the population when accompanied by a sufficient testing capacity or when the testing policy prioritises symptomatic cases. As user rate increases, prevalence of infection decreases. With that, when symptomatic cases are not prioritised for testing, a high rate of app users can generate an extensive increase in the demand for testing, which, if not met with adequate supply, may render the app counterproductive. This points to the crucial role of an efficient testing policy and the necessity to upscale testing capacity.

At the time of writing Coronavirus Disease 2019 (COVID-19) has caused just over 1,200,000 confirmed deaths in 216 countries worldwide ${ }^{1}$. In the absence of an effective treatment, let alone a vaccine, the only possible mitigation strategies are non-pharmaceutical ${ }^{2}$. In the initial phase of the pandemic lockdown measures, implemented with various degrees of firmness in most countries, have proven effective in containing the epidemic ${ }^{3}$ and reducing the basic reproduction number $R_{0}$ to less than 1 . However, these measures cannot be sustained for a prolonged period of time, as the economic damage inflicted on workers, enterprises and governments would be irreparable. Therefore, a second phase of containment has followed, in which lockdown measures are lifted and different mitigation strategies are required, namely case isolation, tracking and contact-tracing, which are rooted in the established epidemiological toolkit ${ }^{4}$. These are particularly crucial in the context of a disease that is transmitted by a large proportion of a-symptomatic and pre-symptomatic individuals, as studies of early outbreaks in China and Italy have indicated ${ }^{5-8}$. However, manual contact tracing can be a time-consuming and inefficient exercise, since models show that the probability of epidemic control decreases rapidly when not enough cases are ascertained via contact-tracing before the onset of symptoms. Technology-based solutions have been proposed to automatise the tracking process, in the form of contact tracing smartphone apps. Exploiting the Bluetooth Low Energy technology, these applications can trace other smartphones coming into close contact for a period of time compatible with potential infection transmission ${ }^{10}$. Once an individual who uses the app discovers having been infected, all smartphones who have come into close contact receive a notification, signalling the potential exposure. Intense debate followed in the scientific community and among the public on the risks, especially to privacy, and effectiveness of the app-based solution ${ }^{11}$. A number of studies, using a range of methodologies, tried to establish the optimal adoption rate and the other necessary measures (such as social distancing and testing) required for the app to be effective in containing or suppressing the epidemic ${ }^{12}$. Analytical mathematical models show a generally optimistic stance, suggesting that instantaneous contact tracing, such as that afforded by the app-based solution, can lead to epidemic control ${ }^{13}$, if at least $60 \%$ adoption rate of the app is attained ${ }^{14}$.

Alternatively, other models highlight possible unintended consequences of the mitigation strategies. For example, adopting the app with an inadequate number of tests available may lead to epidemic control, but at the price of an unrealistic number of people having to isolate ${ }^{15}$. Another model ${ }^{16}$, still in the development phase, seems to suggest that the app might not be effective at all, and other measures could be preferable, such as a random testing policy. 


\begin{tabular}{|c|c|c|c|}
\hline Type of contact & Frequency of encounters & No. of contacts per encounter & Transmission probability $(\beta)$ per contact \\
\hline Household & Daily & All household members & $\beta_{c}$ \\
\hline School & 5 days per week & $50 \%$ of the class & $\beta_{k}=\beta_{c} \times 0.5$ \\
\hline Friendship/Acquaintance & $\begin{array}{l}\text { Daily (age }<65 \text { years); } 3.5 \text { days per week (age }>65 \\
\text { years) }\end{array}$ & $1-10 \%$ of their friends & $\beta_{c}$ \\
\hline Relations & 2 days per week & One relative per household & $\beta_{c}$ \\
\hline Workplace & 5 days per week & All close colleagues and one from other colleagues & $\beta_{c}$ \\
\hline Workplace (public facing) & 5 days per week & $\begin{array}{l}\text { Random contacts are drawn from a Poisson distri- } \\
\text { bution Pois }\left(\lambda_{W}\right) \lambda_{W}=3 \mathrm{p} \times \text { zone }_{-} \text {population }\end{array}$ & $\beta_{r}=\beta_{c} \times 0.1$ \\
\hline Random & $\begin{array}{l}\text { Daily (age }<65 \text { years); } 3.5 \text { days per week (age }> \\
65 \text { years) }\end{array}$ & $\begin{array}{l}\text { Random contacts are drawn from a Poisson distri- } \\
\text { bution Pois }\left(\lambda_{R}\right) \lambda_{R}=\mathrm{p} \times \text { zone } e_{-} \text {population }\end{array}$ & $\beta_{r}=\beta_{c} \times 0.1$ \\
\hline
\end{tabular}

Table 1. Contact type and transmission probability for social network based and random encounters.

Here we present an agent-based model designed to explore the effectiveness of the app in containing or suppressing the epidemic. The model simulates the complex interplay between: (1) the portion of the population that uses the app, (2) the availability of testing, and (3) behavioural factors, such as the willingness to comply with self-isolation instructions. The model explicitly simulates realistic interactions and examines the different policy options on the table. Our modelling approach tries to balance the competing needs for flexibility and scalability, while retaining as much complexity and descriptiveness as possible.

\section{Methods: an agent-based model of COVID-19 transmission and mitigations}

Modelling the effectiveness of mitigation strategies requires that we simulate them in parallel with the spread of the disease itself. Building on the principles of the SEIR approach, an agent-based model (ABM) was developed, simulating the spread of COVID-19 within the population of an urban area. Since the virus is transmitted through contacts between infected and susceptible individuals, in order to understand the dynamics of the spread it is essential to represent the multiple social networks that connect individuals within a population and determine patterns of contacts. A major weakness of traditional compartmental infection models is their aggregated nature. These models divide the population into homogeneous groups (compartments) in accordance with the state of the disease (SEIR), and assume disease transmission to occur as the infected group mixes with the susceptible group at certain rates ${ }^{17}$. These models' assumptions do not account for the heterogeneity that exists between individuals within the groups, and simplify the complexity of contact patterns, which take place in social networks that are important to understanding the course of an epidemic ${ }^{18}$.

Our ABM intends to bridge this gap by modelling more realistic contact patterns that take place among heterogeneous agents interacting within a social network. Central to the agent-based approach is that each agent is represented in the model individually, with their specific characteristics (such as age and sex) and behaviour. Furthermore, interactions between agents are explicitly simulated.

Agents' social networks and daily contacts. We generate a synthetic population of circa 103,000 agents, derived from the $2011 \mathrm{UK}^{\mathrm{Census}}{ }^{19}$, including household type, gender and age within geographical zones (Detailed Characteristic Sector 2011). The population represents the city of Glasgow, Scotland. A multilayered social network links agents within the following social structures:

- A household structure is created as follows: individuals who belong to households classed with the same type in the Census, and reside in the same locale, are linked together on the basis of age difference; single people below the age of 20 are assumed to live at home with one or two parents and siblings. Single people above the age of 20 are assumed to live independently, with a certain proportion co-habiting. Links of type 'household' are built among these agents.

- Family relatives who don't live in the same household (i.e. grandparents) are linked together.

- Several workplace sites are created, based on the distribution of workplace sizes in the city of Glasgow ${ }^{20}$. Active working-age agents are distributed among workplaces and linked to all co-workers at the same site as well as to a subset of colleagues who are assumed to be in closer, more frequent contact. Out of the working-age population, $13 \%$ of agents are assigned to customer-facing employment ${ }^{21}$, experiencing frequent contact with random agents of the population during work.

- A friendship network links agents over 14 years of age, generated following the Barabasi-Albert model ${ }^{22}$ so that a scale-free network is produced, characterised by variation in number of friends per agent, with a median of 14 friends per agent, skewed towards similar age.

- Children between 6-17 years of age also belong to classes of maximum 30 children of the same age from the same zone and are linked together as classmates.

Using the social network we simulate daily contacts that take place between individuals within a population. Each type of social domain (household, workplace/school, friends, relatives and random contacts) is defined by frequency of encounters and the number of contacts per encounter (Table 1). Agents come into contact with all household members daily, and meet with relatives twice per week. They attend the workplace and school 5 days 


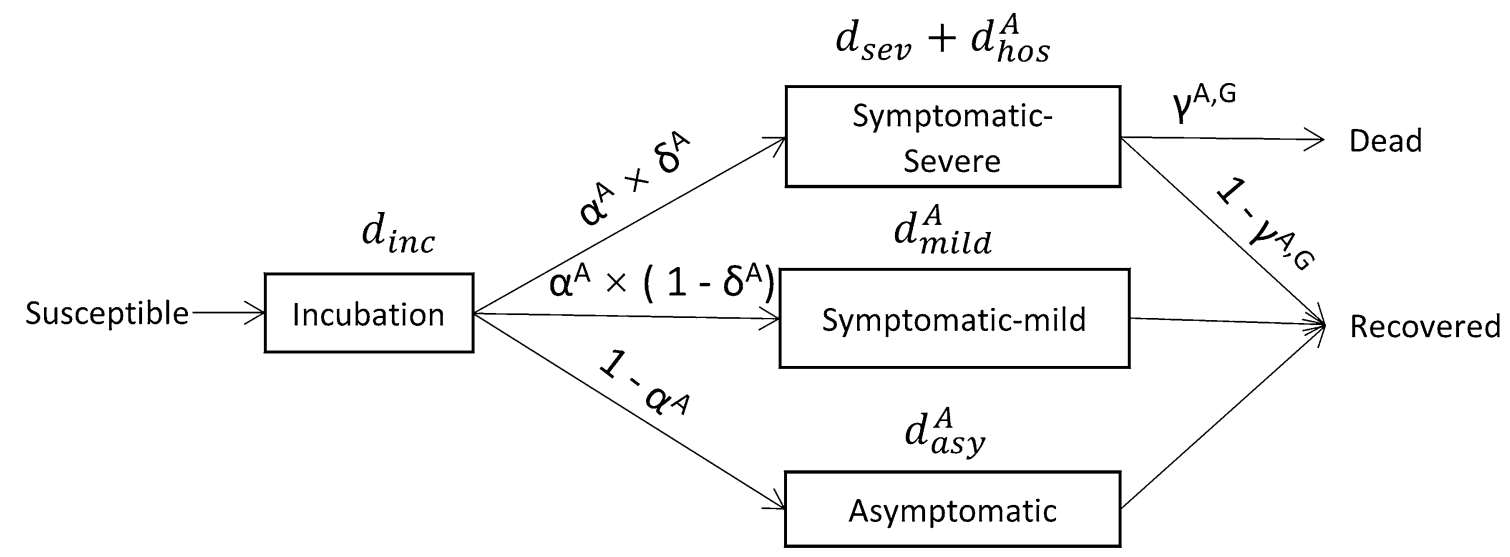

Figure 1. Disease progression. Rectangles represent states of the disease and arrows the transition between states. $d_{\text {state }}^{A}$ denotes the duration of the disease state given the age $A$ of the agent. Agents with severe symptomatic disease spend $d_{\text {sev }}$ days at home before being admitted to the hospital for a duration of $d_{\text {hos }}^{A}$ days. Transition between disease states occurs with age dependent probabilities; where $\alpha^{A}$ and $1-\alpha^{A}$ denote the probability of an agent of age $A$ being symptomatic and asymptomatic, respectively. $\delta^{A}$ denotes the probability for a symptomatic agent to progress into severe disease; $\gamma^{A, G}$ denotes the probability of a severely ill agent of age $A$ and gender $G$ to die. For details of parameter values see: Supplementary Table S1.

per week. For school, we simply assume that during a day, agents have contacts with half of their classmates, which are randomly selected. For the workplace, agents have contacts with a group of close colleagues and with one randomly selected co-worker. In this way workplace employees are clustered in small groups having daily contacts (representing colleagues working in proximity) but also interact less frequently with others in the larger workplace. As for social encounters, it is highly difficult to define a typical number of friends. Here, we wish to account for the heterogeneity that exists between highly socially active individuals, having many friendship ties, and less active ones, having only few social ties. Therefore, for each meeting the number of contacts is randomly selected between 1 friend to $10 \%$ of the agent's friendship ties. Furthermore, we assume that elderly agents have a reduced frequency of social encounters ${ }^{23,24}$. As for random contacts with strangers, we assume the number of contacts is a proportion $p$ of the size of the area of residence (zone size range between 200-2700, with a mean of 1100 agents). Hence, each day agents have random contacts with $p=1 \%(\sim 11$ contacts $)$ of the population residing in their zone, and public-facing workers have contacts with $3 p=3 \%$ ( $\sim 33$ contacts). To reflect daily variability in encounters, the number is sampled from a Poisson distribution. The emerging distribution of daily contacts in the model resembles the shape of the distribution derived from a UK population contact survey (see: "Model calibration and baseline scenario" section). The impact of different contact frequencies on ABM results is furthered explored in a sensitivity analysis (Supplementary Table S2).

Viral transmission. During each day, all infected agents come into contact with the subset of agents in each social environment. For each contact with a susceptible agent the virus may be transmitted with a certain probability (Table 1 ). The probability of transmission during a contact with an agent in the network $\left(\beta_{c}\right)$ is higher than during a random contact with a stranger $\left(\beta_{r}\right)$, as we assume that a contact with a stranger is of shorter duration and reduced closeness, translating in a reduced likelihood of transmission (Table 1). In accordance with evidence that children are less susceptible to COVID- $19^{25}$, we reduce the probability of infection by $50 \%$ for agents under the age of 16 .

Progression of the disease. Once a susceptible agent is infected, she progresses through the various states of the disease (Fig. 1). The progression between disease states and the duration of each state are based on probabilities and durations which are age and gender dependent, as estimated in recent research on COVID-19 patients (Supplementary Table S1). Initially, the disease is in the incubation phase, a stage in which the agent is not infectious. A fraction of the infected agents becomes infectious 1-3 days before the end of the incubation period (pre-symptomatic infection), while others are infectious only at the end of incubation ${ }^{6,26,27}$. To reflect that, during each of the last 3 days of incubation agents have a $25 \%$ probability of becoming infectious. Following the incubation period, agents are either asymptomatic or symptomatic. Asymptomatic agents are able to infect others, but do not feel symptoms, and we assume that after the 3rd day of being infectious, infectiousness declines by $10 \%$ each day that follows ${ }^{28}$. Symptomatic agents with a mild disease are assumed to feel the symptoms, but do not require hospitalisation; severely ill symptomatic agents initially stay at home and are then admitted to the hospital. Once severely ill agents are admitted to the hospital, we assume they do not come into contact with any other agent. The model does not simulate nosocomial infection and always assumes the availability of hospital beds. In accordance with findings, agents stop infecting others after 7-11 days from the onset of symptoms ${ }^{29}$. 


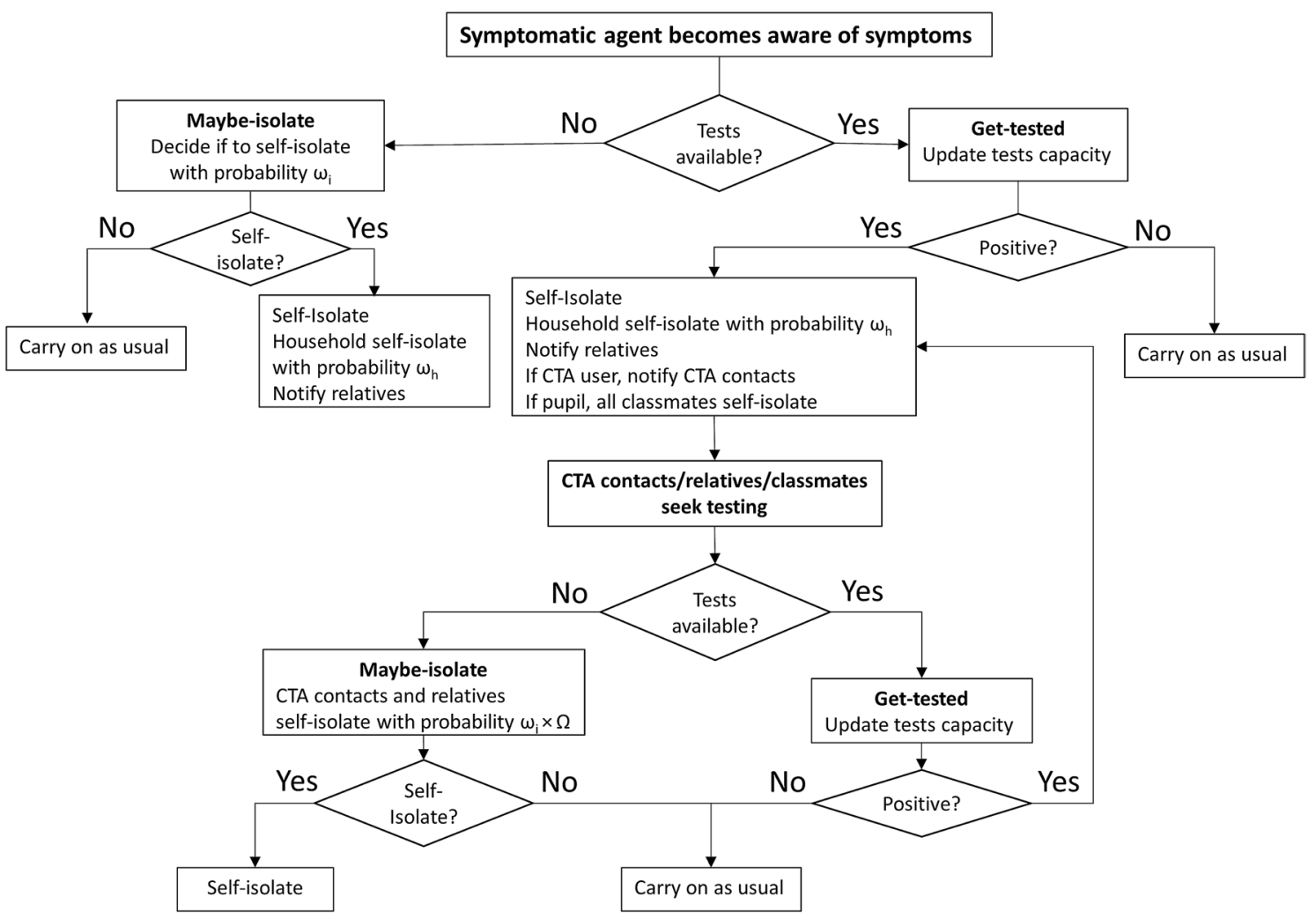

Figure 2. Procedure for testing, self-isolation and CTA notification. Once symptomatic agent $i$ becomes aware of the disease, the agent seeks testing. If tests are available, the agent gets tested. Following a positive result the agent will self-isolate. Household members $h$ of agent $i$ will self-isolate with probability $\omega_{h}$ and relatives will be notified. If the agent is a pupil all classmates will self-isolate and seek testing. If agent $i$ uses the CTA all the recorded contacts will be notified. When tests are unavailable, agent $i$ will self-isolate with probability $\omega_{i}$. In case the agent self-isolates the aforementioned procedure of self-isolation will take place without the notification to CTA contacts. Otherwise the agent will continue as usual. Once CTA user $j$ is notified, agent $j$ will seek testing. If testing is unavailable, agent $\mathrm{j}$ may self-isolate with probability $\omega_{j} * \Omega$.

Mitigation strategies: testing, contact tracing app. The model includes two types of mitigation tools to track and trace infected agents: the contact tracing app (CTA) and COVID-19 detection tests. The CTA is distributed among a fraction of the population aged over 14. It stores in memory the ID of all other CTAs it came into contact with over the course of the previous 10 days. Infected agents who were tested positive can use the CTA to notify their contacts of possible exposure.

Symptomatic agents who seek testing are assumed to get tested between 1 and 3 days after the onset of symptoms, and results are determined within a day. We assume that a fixed number of tests is available: as agents are tested the stocks decrease and restocking takes place daily. Agents seek testing when: (a) they feel symptoms, (b) they are notified of possible exposure to an infected agent. A notification is made to exposed agents when: (a) an agent feels symptoms or receives a positive test result and alerts all relatives; (b) a pupil tests positive and all classmates are made aware and quarantined; (c) a CTA user receives a positive test result and the app notifies all recorded contacts (Fig. 2).

To reflect the impact of influenza-like illness (ILI) on the testing system, we assume that over the course of any given week, $3.5 \%$ of the population experience ILI $^{30}$, of which $30 \%$ will seek COVID-19 testing. While these agents test negative, they contribute to the depletion of tests.

Agents' adherence to self-isolation instructions. When agents self-isolate all their social ties are removed, except for household ties, as they are assumed to self-isolate at home. It is likely that some precautions are put in place between the infected agent and her household members, therefore we assume a $30 \%$ reduction in the probability of transmission within the household.

Without the certainty that testing provides, surveys suggest that not all individuals will comply with selfisolation guidelines, both when feeling symptoms or when notified by the CTA ${ }^{31}$. We denote a parameter $\omega_{i}$ representing the probability of agent $i$ to self-isolate when feeling symptoms of COVID-19. We also assume that agents who are notified by the CTA, but do not feel any symptoms, are less likely to self-isolate (than if they had symptoms) without testing. Therefore, their probability to self-isolate is reduced by factor $\Omega$, where $0<\Omega<1$. In the model, the probability of self-isolation varies between agents with mean $\omega=70 \%$. Figure 2 presents the algorithm triggered once an agent becomes aware of her symptoms. The procedure triggers a chain of actions performed by symptomatic agents that involves testing (if available), deciding whether to self-isolate and notifying 

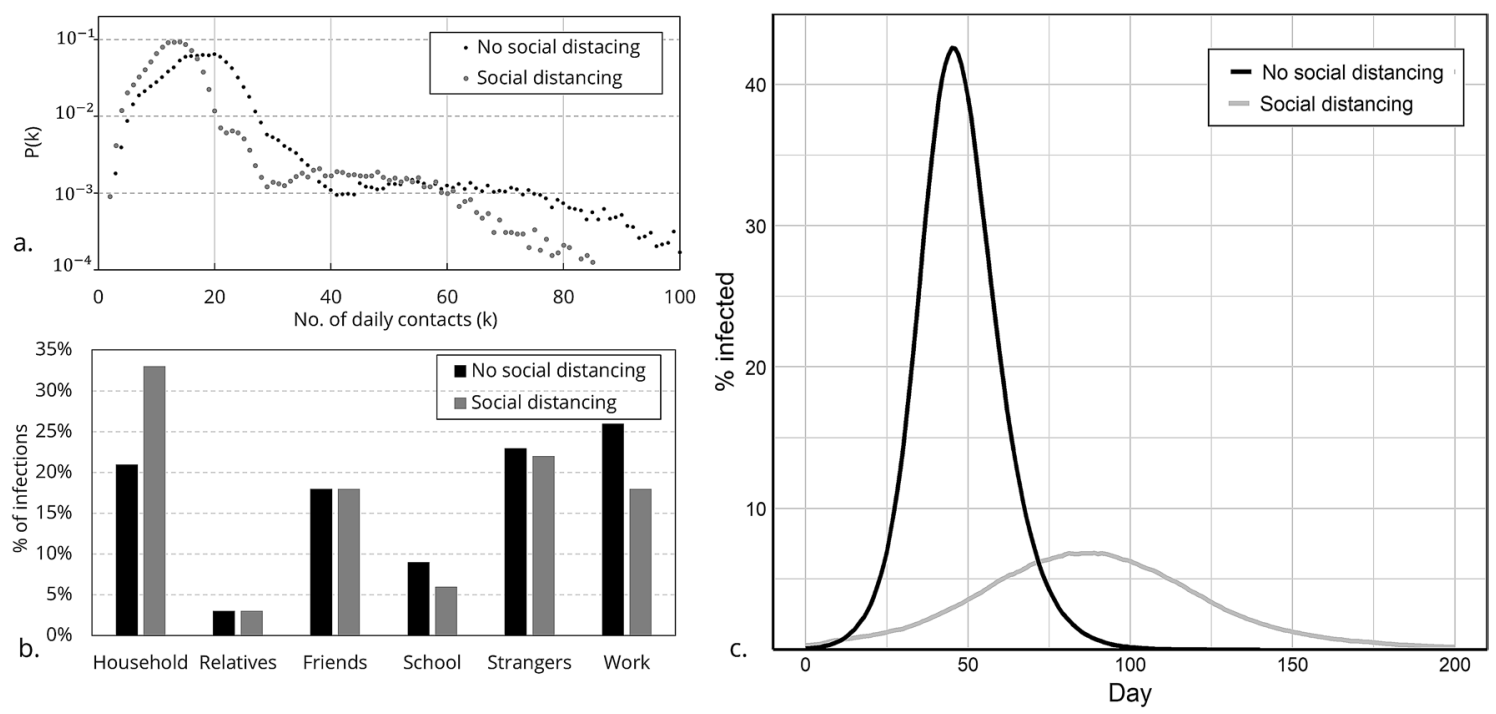

Figure 3. Distribution of daily contacts and epidemic dynamics, for scenarios with and without social distancing. (a) Distribution of number of daily contacts. (b) Distribution of infection sources by type of contact. (c) Infection prevalence by day, from beginning to end of the epidemic.

relatives and CTA contacts. Following that, exposed agents who were notified perform similar actions. For a pseudo code of the main model functions see: Supplementary Information.

\section{Model calibration and baseline scenario}

The initial scenario reproduces a 'business as usual' situation with no mitigation in place, with contact frequencies as specified in Table 1 . To verify the contact patterns generated by the ABM, we compared the properties of the distribution of agents' daily contacts generated by the model to a distribution of contacts derived from a survey conducted in the $\mathrm{UK}^{32}$. The distribution of daily contacts generated by the ABM holds a similar shape to the distribution in the survey. It is characterised by a lognormal body where most contacts are between 5-25, and a long tail of higher number of contacts with lower frequencies (Fig. 3a). The mean and standard deviation of number of daily contacts are 20 and 13 , respectively.

To calibrate the model, we tested a range of transmission probability $\left(\beta_{c}\right)$ values to generate the basic reproduction number of $R_{0} \sim 2.8$ in the initial three weeks of the epidemic, as estimated for the $\mathrm{UK}^{33}$. The best fit was achieved for $\beta_{c}=8 \%$, and $\beta_{r}=0.8 \%$.

After establishing the initial scenario, we simulate the post-lockdown situation expected in several countries, in which most restrictions are lifted but citizens are still encouraged to work from home when possible, limit social interactions, maintain physical distancing and wear face masks in public. Therefore, in this scenario we assume 3 days attendance per week at workplaces and schools; and a reduction of $30 \%$ in contacts in schools, with strangers, as well as the frequency of social meetings (within the 'friendship' network). The contact distribution in this scenario is presented in Fig. 3a. The mean number of daily contacts is reduced from 20 to 14 . In addition, transmission probability for contacts outside of the household is reduced by $30 \%\left(\beta_{c}=5.6 \%\right.$ and $\left.\beta_{r}=0.56 \%\right)$ to reflect measures such as face mask usage, social distancing and increased hygiene, all of which reduce the likelihood of viral transmission. We refer to this situation as our baseline scenario. The reproduction number in this scenario comes down to 1.5. Comparing the scenarios, when social distancing is practised, the proportion of infected agents at the peak of the epidemic is significantly reduced from 43 to $7 \%$ (Fig. 3c). The distribution of the sources of infection also varies, as the proportion of infections originating in workplaces and schools is reduced and the household becomes the predominant locus of transmission (Fig. 3b).

\section{Experimental design}

The core of our study explores the introduction of the CTA and the availability of testing into the baseline scenario of social distancing. We simulate the impact on viral spread of various combinations of: (1) proportion of CTA users in the population; (2) levels of testing capacity; (3) levels of compliance with self-isolation on the part of CTA users; (4) testing policy. Table 2 summarises the parameter combinations explored in the model. Overall, we simulated 140 scenarios, each repeated 20 times to account for uncertainty in the results due to the stochasticity embedded in the model.

\section{Results: effectiveness of smartphone-based track-and-trace policies}

To evaluate differences in viral diffusion under alternative parameter combinations, for each simulated scenario we plot the overall proportion of the population infected at the peak and throughout the whole course of the epidemic. We use the baseline scenario of no tests and no app as point of reference to better understand the impact of the mitigations that are introduced. 


\begin{tabular}{|l|l|l|}
\hline Parameter & Value in experiments & Description \\
\hline CTA users & $0,20,40,60,80$ & \% population over age 14 using CTA \\
\hline Testing capacity & $0,0.5,1,1.5,3,6$, unlimited & $\begin{array}{l}\text { Maximum \% of population that can be tested per week (reflective of UK policy announce- } \\
\text { ments of 200,000 tests per day, corresponding to 2\% of the population per week) }\end{array}$ \\
\hline Compliance of CTA users & Low compliance: $\Omega=0.5$ High compliance: $\Omega=0.9$ & $\begin{array}{l}\text { Probability to self-isolate for CTA user without testing decreases by } \Omega \text { : probability to self- } \\
\text { isolate }=\omega_{i} * \Omega\end{array}$ \\
\hline Testing policy & a) Priority to symptomatic agents b) No priority & $\begin{array}{l}\text { a) Each day symptomatic agents are tested, only then CTA users b) Agents are tested on basis } \\
\text { of first come first tested }\end{array}$ \\
\hline Initial conditions & $7 \%$ recovered from the virus; $300(0.3 \%)$ agents infected & Reflects UK estimates ahead of lifting lockdown \\
\hline
\end{tabular}

Table 2. Parameters used in simulation experiments.
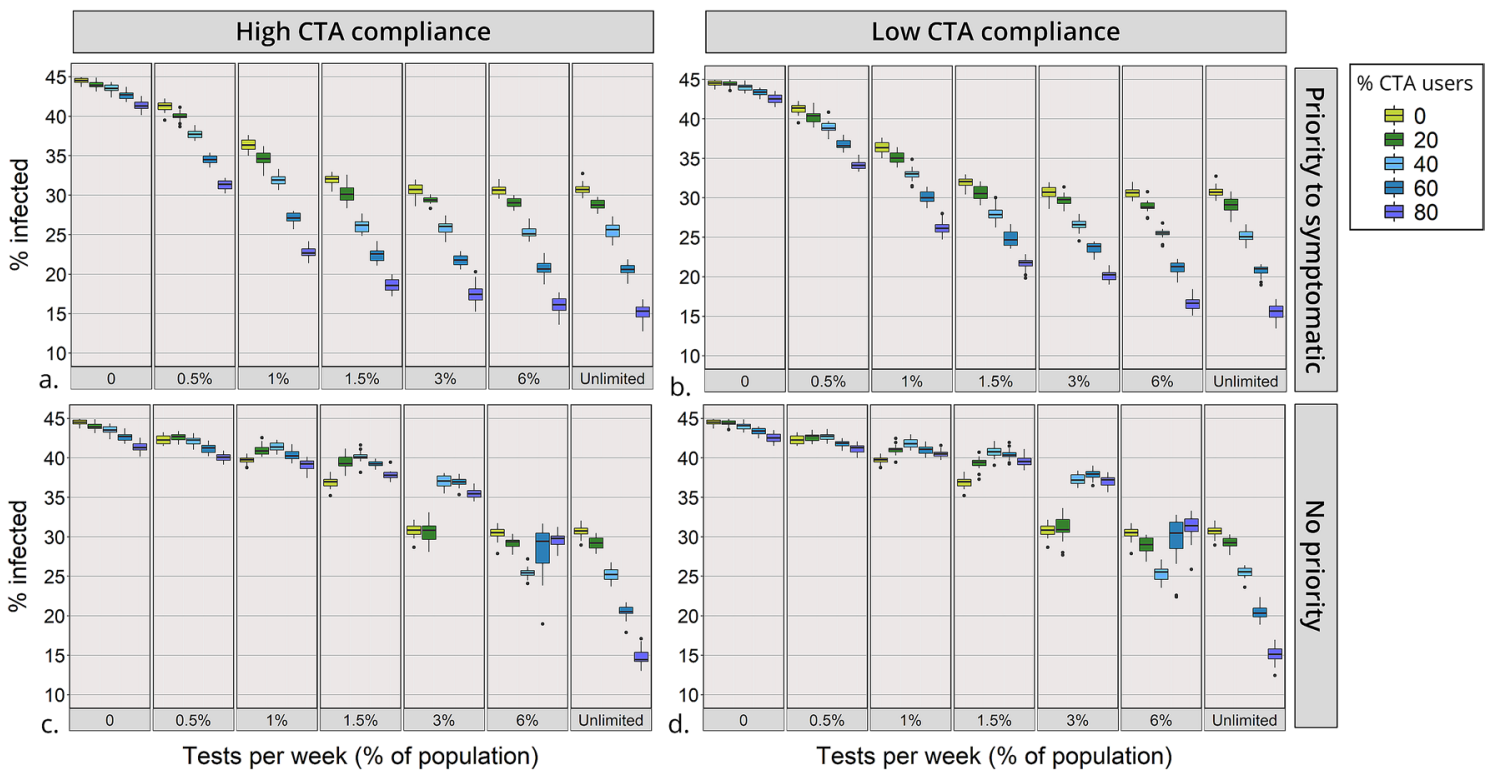

Figure 4. Percentage of the population infected during the course of epidemic. Scenarios vary by testing capacity ( $\mathrm{x}$-axis) and percentage of CTA users (boxplot's colour). Diagrams are organized by testing policy and compliance with self-isolation of CTA users: (a) High compliance and priority for testing symptomatic cases; (b) Low compliance and priority for testing symptomatic cases; (c) High compliance and no priority for testing symptomatic cases; (d) Low compliance and no priority for testing symptomatic cases. The boxplots show the median and interquartile range of multiple simulation runs.

Testing without tracing. When testing is the only mitigation used (with no CTA), as testing capacity increases from 0 to $3 \%$, overall infections decreases from 44 to $31 \%$ (Fig. 4 ) and infections at the peak are reduced by $59 \%$ (Fig. 5). A further increase in testing capacity above $3 \%$ does not result in a further decrease in infections. This can be explained by the prevalence of infected agents per day, which reaches $3 \%$ of the population at the peak of the epidemic (Fig. 6a, light green line). Since around $50 \%$ of the infected are symptomatic, a testing capacity of $3 \%$ appears as sufficient to test all the symptomatic COVID-19 cases, other contacts who were notified (such as relatives and classmates) as well as the portion of influenza-like illness cases that seek testing. Thus, testing increases compliance with self-isolation which in turn reduces transmission.

Introducing the CTA with a testing policy that prioritises symptomatic cases. Once the CTA is introduced, CTA users who are notified of having been in contact with an infected agent seek testing. When symptomatic agents are prioritised for testing, as the proportion of CTA users increases, overall infections throughout the epidemic decrease (Fig. 4a,b), and so do infections at the peak of the epidemic (Fig. 5a,b), irrespective of testing capacity. Note that some reduction in overall infections is observed as CTA adoption rates increase even in the scenario with no testing; in this case CTA notifications are emitted only by a fraction of app users who were severely ill and diagnosed in the hospital. Moreover, a synergy exists between testing and the CTA, resulting in a larger reduction in the spread of the virus, reflected in a decrease in infections both overall, and at the peak of the epidemic. The decrease is most substantial when testing is not limited. For example, when $80 \%$ of the population uses the CTA, the percentage of the population infected throughout the epidemic decreases from 45 to $15 \%$ (Fig. 4 a,b) and cases at the peak of the epidemic reduce by $89 \%$ (Fig. 5a,b). Moreover, for intermediate levels of CTA adoption (40-60\%) and testing capacity (1.5-3\%), overall infections decrease to $22-27 \%$ of the population and cases at the peak reduce by $70-85 \%$, depending on the scenario (Figs. $4 a, b ; 5 a, b)$. 

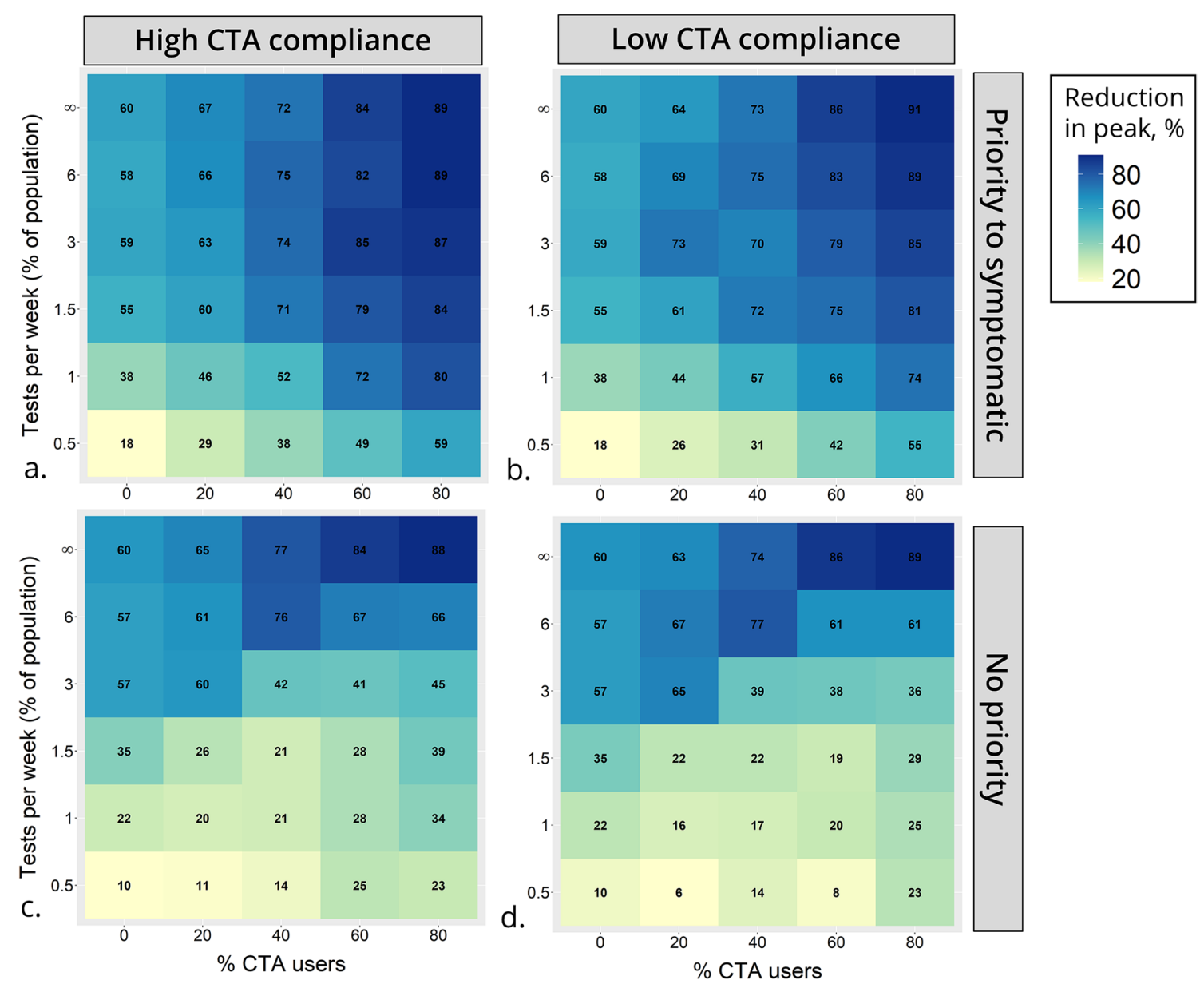

Figure 5. Reduction in infection prevalence at the peak of the epidemic. Reduction is relative to the peak of the epidemic in the baseline scenario and measured as percent reduction of this value. Scenarios vary by percent of CTA users (x-axis) and testing capacity (y-axis) and organised by testing policy and compliance with selfisolation of CTA users: (a) high compliance and priority for testing symptomatic cases; (b) low compliance and priority for testing symptomatic cases; (c) high compliance and no priority for testing symptomatic cases; (d) low compliance and no priority for testing symptomatic cases.

CTA with no priority to test symptomatic cases. When the testing capacity is restricted and symptomatic agents are not prioritised for testing, an increase in the proportion of CTA users does not always lead to a decrease in infections (Fig. 4c,d). This is observed for all testing capacities between 0 and $6 \%$. In the case of a 3\% testing capacity, CTA adoption rates of $40-80 \%$ result in substantially more infections than the scenario with no CTA users, both for high and low compliance with the CTA. This somewhat counterintuitive effect is explained by an inefficient testing policy. If symptomatic agents are not prioritised, they are as likely to be tested as those notified by the app, the majority of which are not infected. However, the increased demand for testing generated by CTA users who receive notifications depletes the stocks and prevents several infected cases from being detected. This phenomenon is apparent when comparing the 'efficiency' of the two testing policies (Fig. 6c,d). When symptomatics are prioritised, the proportion of positive tests is higher for any given rate of CTA adoption, even though the prevalence of infection is lower than in the no priority scenario.

In general, the model suggests that the adoption of the CTA triggers two competing dynamics. On one hand, it informs agents and leads some of those infected to isolate even in the absence of a test: a crucial outcome, given the high proportion of a-symptomatic cases. On the other hand, it leads to a large and somewhat 'inefficient' depletion of tests, as several uninfected agents would likely seek testing after a notification from the app. The model shows that this counterproductive effect can be mitigated either with a testing policy that prioritises symptomatics cases, or with an overall increase in testing capacity. The scenarios with unlimited tests always show a substantial advantage in using the CTA.

CTA users' compliance with self-isolation. When compliance with self-isolation of CTA users is high, overall infections throughout the epidemic are only a few percentage points $(\sim 3-5 \%)$ lower than when compliance level is low. The level of compliance with self-isolation becomes more influential the higher the proportion of CTA users, and at lower levels of testing capacity (Fig. 4). E.g. in a scenario with $1 \%$ testing and $80 \%$ CTA users, overall infections reach $23 \%$ when compliance is high and $27 \%$ when compliance is low. The peak of the 
Priority to symptomatic
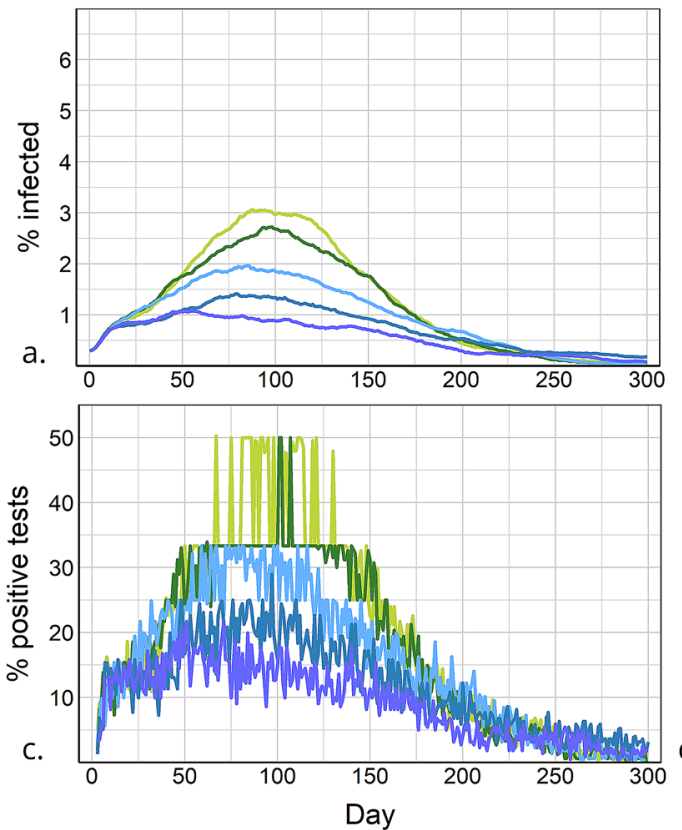

No priority
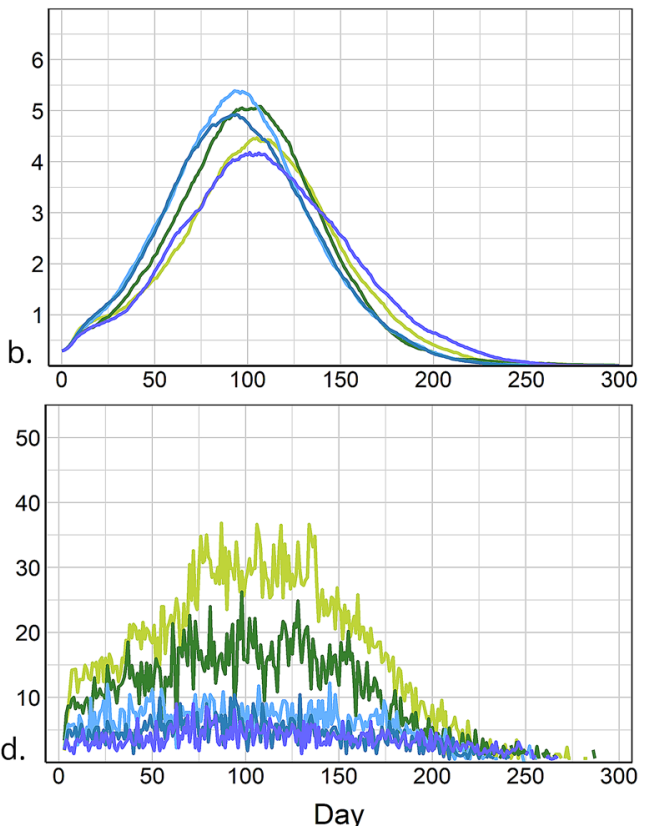

Figure 6. Infection prevalence and positive tests for a testing policy with and without priority to symptomatic cases. Percentage infected (a) and percentage of positive tests (c) by day, for a testing policy with priority to symptomatic. Percentage infected (b) and percentage positive tests (d) by day, for a testing policy without priority. Percentage of CTA users in the scenario is marked by line colour. In these scenarios: testing capacity = $1.5 \%$ and CTA compliance is high. Note that in the scenarios with no CTA users, although only symptomatic cases are tested, the percentage of positive tests is not $100 \%$ because of cases with influenza-like illness that are also being tested.

epidemic compared to the baseline scenario is reduced by $\sim 80 \%$ when compliance is high and $\sim$ by $74 \%$ when compliance is low (Fig. 5).

\section{Discussion}

The primary aim of this work is to contribute to the understanding of the complexity embedded in the interaction between the circulation of COVID-19 and the mitigations proposed. The results presented above show that app-based contact-tracing has the potential to mitigate the spread of COVID-19 in a social distancing scenario. With that, CTA efficiency in reducing the spread relies on additional elements: testing capacity and management of limited testing resources. When priority for testing is given to symptomatic cases the impact of CTA is linear; the higher the levels of CTA adoption, the more the virus is suppressed. This is true for any level of CTA adoption and any level of testing capability.

A more complex dynamic emerges in the model under limited testing resources when symptomatic cases are not prioritised. Increases in the adoption of the CTA produce a spillover effect whereby the large number of (mostly non-infected) agents notified by the CTA depletes the testing stock and prevents a number of symptomatic agents from being tested. In this case, the CTA may even produce an increase in the infection rate, especially if testing capacity is not sufficiently high relative to the number of CTA users.

These phenomena that emerge in the simulation can offer generalisable policy-relevant insights. First, the model shows that to optimise the contribution of the CTA towards epidemic control an adequate testing capacity has to be in place. This capacity is dependent on the proportion of CTA users in the population. Second, the specific way in which testing resources are managed substantially affects the effectiveness of containment. The suggestion is that governments should implement the CTA in parallel with a substantial increase in testing capacity, and accurately plan the details of the testing policy, keeping in mind that the mere availability of the CTA substantially alters patterns of demand for testing.

One of the aims of the model was to explore the impact of certain behavioural factors: we have shown that, even under conditions of low compliance of CTA users towards self-isolation, a significant decrease in the spread of the virus is achieved. Moreover, compliance with self-isolation can be enhanced by increasing the availability of testing to provide more certainty to exposed individuals regarding their infection state. Recent findings suggest that providing people with assurances about their livelihoods, by means of financial compensation, will increase compliance with self-isolation ${ }^{34}$.

As we demonstrated, in the more optimistic scenarios overall infections throughout the course of the epidemic are reduced to a third of the baseline scenario. The model shows that, when accompanied by social distancing, CTA and testing are likely to reduce the spread of the epidemic and contribute significantly to lowering the epidemic peak. This is especially crucial for the functioning and manageability of the healthcare system until a 
vaccine and other pharmaceutical treatments are developed. Furthermore, the model suggests that the effectiveness of the CTA is higher in situations of higher viral circulation (Sensitivity analysis, Supplementary Information). This potentially increases its importance in controlling the epidemic at the post-lockdown stage, where reopening of economic activity inevitably leads to higher infection rates.

Limitations. The unprecedented nature of technology-based contact tracing, and the novelty of the disease itself, make it difficult to include, parametrise and validate every aspect of the model based on hard evidence, not least because such evidence often does not yet exist. As for any modelling exercise, the generated results rely on the model's assumptions that simplify reality. We based the disease course parameters on emerging evidence as specified in Supplementary Table S1, while the individual level transmission parameters were calibrated to match the aggregated reproduction number $\left(R_{0}\right)$ reported in the UK (Table 1$)$. Validation of the contact patterns emerging in the model was based on a comparison with a UK-based survey (Fig. 3). With that, even with proper calibration of the ABM to reported infection rates $\left(R_{0}\right)$ it is possible that a different combination of individual based contact patterns and transmission rates occurring in different social environments could produce similar infection rates on the aggregated level ${ }^{35}$. In such a case, the relative contribution of different social domains to the spread may differ and affect the extent to which CTA is employed. To address this issue, a sensitivity analysis for parameters affecting the viral spread is offered in the Supplementary Information section. The analysis shows that while the overall proportion of population infected may vary across parameter values, the findings regarding the impact of the CTA remain consistent for all scenarios. Another potential factor not implemented in our model is adoption rate differentials among demographic groups: the impact of such heterogeneity is still to be explored. A final caveat relates to our assumptions that the CTA functions "perfectly" (no technical malfunctions or notification delays) and that test results are accurate and returned within one day. In reality, shortcomings in these matters may impair the contact tracing strategy.

\section{Conclusions}

To conclude, we maintain that smartphone-based contact-tracing is a viable epidemic mitigation strategy, worth pursuing on the part of governments. The model suggests that, as larger fractions of society adopt the CTA, the spread of the virus is increasingly reduced, and, therefore, the benefits extend to the wider population. In principle, the CTA offers speed and cost efficiencies that can complement and extend traditional manual contacttracing methods. In our view, the idea of technology-based contact-tracing should not be dismissed, especially not on the grounds that it may widen inequality by penalising those with limited access to the technology, or fail protect the elderly population who is less likely to adopt it. Paradoxically, users of the CTA do not benefit directly from doing so, since it only operates when an individual may already have been exposed to the virus ${ }^{36}$. While the CTA operates on a personal level by informing individuals of a possible risk of exposure, its general impact is at the societal level. By tracking exposed individuals and informing them to seek testing and self-isolate, transmission chains are interrupted, the spread of the virus is reduced, and so is the likelihood of infection for the whole population, including those who are not using the CTA.

\section{Code availability}

The full source code and datasets employed are open source and maintained at the following address: http:// github.com/harrykipper/covid.

Received: 28 July 2020; Accepted: 30 November 2020

Published online: 17 December 2020

\section{References}

1. World Health Organization. Coronavirus disease pandemic. https://www.who.int/emergencies/diseases/novel-coronavirus-2019 (2020).

2. Ferguson, N. M. et al. Report 9: impact of non-pharmaceutical interventions (NPIs) to reduce COVID-19 mortality and healthcare demand. Technical report March (2020). https://doi.org/10.25561/77482.

3. Flaxman, S., Mishra, S., Gandy, A. \& Al, E. Report 13: estimating the number of infections and the impact of non-pharmaceutical interventions on COVID-19 in 11 European countries. Technical report March (2020). https://doi.org/10.25561/77731.

4. Fraser, C., Riley, S., Anderson, R. M. \& Ferguson, N. M. Factors that make an infectious disease outbreak controllable. Proc. Nat. Acad. Sci. USA 101, 6146-6151. https://doi.org/10.1073/pnas.0307506101 (2004).

5. Lavezzo, E. et al. Suppression of COVID-19 outbreak in the municipality of Vo, Italy. medRxivhttps://doi. org/10.1101/2020.04.17.20053157 (2020).

6. Gandhi, M., Yokoe, D. S. \& Havlir, D. V. Asymptomatic transmission, the Achilles' heel of current strategies to control Covid-19. N. Engl. J. Med. 382, 2158-2160. https://doi.org/10.1056/NEJMe2009758 (2020).

7. Bai, Y. et al. Presumed asymptomatic carrier transmission of COVID-19. JAMA 323, 1406. https://doi.org/10.1001/jama.2020.2565 (2020).

8. Tong, Z.-D. et al. Potential presymptomatic transmission of SARS-CoV-2, Zhejiang Province, China, 2020. Emerg. Infect. Dis. 26, 1052-1054. https://doi.org/10.3201/eid2605.200198 (2020).

9. Hellewell, J. et al. Feasibility of controlling COVID-19 outbreaks by isolation of cases and contacts. Lancet Glob. Health 8, e488e496. https://doi.org/10.1016/S2214-109X(20)30074-7 (2020).

10. Zastrow, M. Coronavirus contact-tracing apps: can they slow the spread of COVID-19?. Naturehttps://doi.org/10.1038/d4158 6-020-01514-2 (2020).

11. Sweeney, Y. Tracking the debate on COVID-19 surveillance tools. Nat. Mach. Intell. 2, 301-304. https://doi.org/10.1038/s4225 6-020-0194-1 (2020).

12. Braithwaite, I., Callender, T., Bullock, M. \& Aldridge, R. W. Automated and partly automated contact tracing: a systematic review to inform the control of COVID-19. Lancet Dig. Health 2, e607-e621. https://doi.org/10.1016/s2589-7500(20)30184-9 (2020).

13. Ferretti, L. et al. Quantifying SARS-CoV-2 transmission suggests epidemic control with digital contact tracing. Science 6936, eabb6936. https://doi.org/10.1126/science.abb6936 (2020). 
14. Fraser, C. et al. Digital contact tracing: comparing the capabilities of centralised and decentralised data architectures to effectively suppress the COVID-19 epidemic whilst maximising freedom of movement and maintaining privacy. Introduction 2 (2020).

15. Firth, J. A. et al. Combining fine-scale social contact data with epidemic modelling reveals interactions between contact tracing, quarantine, testing and physical distancing for controlling COVID-19. medRxiv. https://doi.org/10.1101/2020.05.26.20113720 (2020).

16. Assocc - agent-based social simulation of the coronavirus crisis. https://simassocc.org/scenario-effect-of-tracktrace-apps/ (2020).

17. Chitnis, N., Hyman, J. M. \& Valle, S. Y. D. Mathematical models of contact patterns between age groups for predicting the spread of infectious diseases. Math. Biosci. Eng. 10, 1475-1497. https://doi.org/10.3934/mbe.2013.10.1475 (2013).

18. Nielsen, B. F., Sneppen, K., Simonsen, L. \& Mathiesen, J. Heterogeneity is essential for contact tracing. medRxivhttps://doi. org/10.1101/2020.06.05.20123141 (2020).

19. Office for National Statistics. 2011 UK census aggregate data. http://dx.doi.org/10.5257/census/aggregate-2011-1 (2016).

20. Scottish Government. Statistics.gov.scot: business, enterprise and energy. https://statistics.gov.scot/resource?uri=http://statistics .gov.scot/def/concept/folders/themes/business-enterprise-and-energy (2019).

21. Office for National Statistics. Emp04: employment by occupation. https://www.ons.gov.uk/employmentandlabourmarket/peopl einwork/employmentandemployeetypes/datasets/employmentbyoccupationemp04 (2018).

22. Barabási, A.-L. \& Albert, R. Emergence of scaling in random networks. Science 286, 509-512. https://doi.org/10.1126/scien ce.286.5439.509 (1999).

23. Kwok, K. O. et al. Social contacts and the locations in which they occur as risk factors for influenza infection. Proc. R. Soc. B Biol. Sci. 281, 20140709 (2014).

24. Mossong, J. et al. Social contacts and mixing patterns relevant to the spread of infectious diseases. PLOS Med. 5, 1-1. https://doi. org/10.1371/journal.pmed.0050074 (2008).

25. Davies, N. G. et al. Age-dependent effects in the transmission and control of COVID-19 epidemics. medRxivhttps://doi. org/10.1101/2020.03.24.20043018 (2020).

26. Lauer, S. A. et al. The incubation period of coronavirus disease 2019 (COVID-19) from publicly reported confirmed cases: estimation and application. Ann. Intern. Med. 172, 577-582. https://doi.org/10.7326/M20-0504 (2020).

27. Huang, L. et al. Rapid asymptomatic transmission of COVID-19 during the incubation period demonstrating strong infectivity in a cluster of youngsters aged 16-23 years outside Wuhan and characteristics of young patients with COVID-19: a prospective contact-tracing study. J. Infect. 80, e1-e13. https://doi.org/10.1016/j.jinf.2020.03.006 (2020).

28. Yang, R., Gui, X. \& Xiong, Y. Comparison of clinical characteristics of patients with asymptomatic vs symptomatic coronavirus disease 2019 in Wuhan, China. JAMA Netw. Open 3, e2010182. https://doi.org/10.1001/jamanetworkopen.2020.10182 (2020).

29. MIN, C. O. W. Position statement from the national centre for infectious diseases and the chapter of infectious disease physicians, academy of medicine, singapore: Period of infectivity to inform strategies for de-isolation for covid-19 patients. https://www.ams. edu.sg/view-pdf.aspx?file=media\%5C5556_fi_331.pdf\&ofile=Period+of+Infectivity+Position+Statement $+($ final $)+23-5-20+(\operatorname{logos}$ ).pdf (2020).

30. Public Health England. Flusurvey. https://doi.org/10.1073/pnas.03075061011 (2020).

31. Altmann, S. et al. Acceptability of app-based contact tracing for COVID-19: cross-country survey evidence. SSRN Electron. J.https ://doi.org/10.2139/ssrn.3590505 (2020).

32. Danon, L., House, T. A., Read, J. M. \& Keeling, M. J. Social encounter networks: collective properties and disease transmission. J. R. Soc. Interface 9, 2826-2833. https://doi.org/10.1073/pnas.03075061012 (2012).

33. Stedman, M. et al. A phased approach to unlocking during the COVID-19 pandemic-Lessons from trend analysis. Int. J. Clin. Pract.https://doi.org/10.1111/ijcp.13528 (2020).

34. Bodas, M. \& Peleg, K. Self-isolation compliance in the COVID-19 era influenced by compensation: findings from a recent survey in Israel. Health Aff. (Project Hope) 39, 936-941. https://doi.org/10.1377/hlthaff.2020.00382 (2020).

35. Axtell, R. \& Epstein, J. Agent-based modeling: understanding our creations. Bull. Santa Fe Inst. 9, 28-32 (1994).

36. Rizzo, E. COVID-19 contact tracing apps: the "elderly paradox". Public Health 185, 127. https://doi.org/10.1073/pnas.0307506101 $4(2020)$.

\section{Acknowledgements}

This work was supported by the Medical Research Council [Grant Numbers MC_UU_12017/10 and MC_ UU_12017/14] and Chief Scientist Office [Grant Number SPHSU10]. Both Grants provide 5 years of core research support for JA and SP.

\section{Author contributions}

Both authors conceived and programmed the model, designed experiments, analysed the results, and reviewed the manuscript.

\section{Competing interests}

The authors declare no competing interests.

\section{Additional information}

Supplementary Information The online version contains supplementary material available at https://doi. org/10.1038/s41598-020-79000-y.

Correspondence and requests for materials should be addressed to S.P.

Reprints and permissions information is available at www.nature.com/reprints.

Publisher's note Springer Nature remains neutral with regard to jurisdictional claims in published maps and institutional affiliations. 
(c) (i) Open Access This article is licensed under a Creative Commons Attribution 4.0 International cc) License, which permits use, sharing, adaptation, distribution and reproduction in any medium or format, as long as you give appropriate credit to the original author(s) and the source, provide a link to the Creative Commons licence, and indicate if changes were made. The images or other third party material in this article are included in the article's Creative Commons licence, unless indicated otherwise in a credit line to the material. If material is not included in the article's Creative Commons licence and your intended use is not permitted by statutory regulation or exceeds the permitted use, you will need to obtain permission directly from the copyright holder. To view a copy of this licence, visit http://creativecommons.org/licenses/by/4.0/.

(C) The Author(s) 2020 